\title{
199.
}

\section{NOTE ON THE THEORY OF ELLIPTIC MOTION.}

[From the Philosophical Magazine, vol. xI. (1856), pp. 425-428.]

IF, as usual, $r, \theta$ denote the radius vector and longitude, and $\mu$ the central mass, then the Vis Viva and Force function are respectively

$$
\begin{aligned}
& T=\frac{1}{2}\left(r^{\prime 2}+r^{2} \theta^{\prime 2}\right), \\
& U=\frac{\mu}{r}
\end{aligned}
$$

and writing

$$
\begin{aligned}
& \frac{d T}{d r^{\prime}}=r^{\prime}=p \\
& \frac{d T}{d \theta^{\prime}}=r^{2} \theta^{\prime}=q
\end{aligned}
$$

we have $r^{\prime}=p, \theta^{\prime}=\frac{q}{r^{2}}$, and $T=\frac{1}{2}\left(p^{2}+\frac{q^{2}}{r^{2}}\right)$, whence, putting $H=T-U$, the value of $H$ is

$$
H=\frac{1}{2}\left(p^{2}+\frac{q^{2}}{r^{2}}\right)-\frac{\mu}{r}
$$

and by Sir W. R. Hamilton's theory, the equations of motion are

$$
\begin{aligned}
& \frac{d r}{d t}=\frac{d H}{d p}, \frac{d p}{d t}=-\frac{d H}{d r} \\
& \frac{d \theta}{d t}=\frac{d H}{d q}, \frac{d q}{d t}=-\frac{d H}{d \theta}
\end{aligned}
$$


or substituting for $H$ its value, the equations of motion are

$$
\begin{aligned}
& \frac{d r}{d t}=p, \\
& \frac{d \theta}{d t}=\frac{q}{r^{2}}, \\
& \frac{d p}{d t}=\frac{q^{2}}{r^{3}}+\frac{\mu}{r^{2}}, \\
& \frac{d q}{d t}=0 .
\end{aligned}
$$

Putting, as usual, $\mu=n^{2} a^{3}$, and introducing the eccentric anomaly $u$, which is given as a function of $t$ by means of the equation

$$
n t+c=u-e \sin u,
$$

(so that $\frac{d u}{d t}=\frac{n}{1-e \cos u}$ ), the integral equations are

$$
\begin{aligned}
q & =n a^{2} \sqrt{1-e^{2}}, \\
p & =\frac{n a e \sin u}{1-e \cos u}, \\
r & =a(1-e \cos u), \\
\theta-\sigma & =\tan ^{-1}\left(\frac{\sqrt{1-e^{2}} \sin u}{\cos u-e}\right) ;
\end{aligned}
$$

where the constants of integration $a, e, c, \varpi$ denote as usual the mean distance, the eccentricity, the mean anomaly at epoch, and the longitude of pericentre.

Suppose that $q_{0}, p_{0}, r_{0}, \theta_{0}, u_{0}$ correspond to the time $t_{0}$ ( $q$ is constant, so that $\left.q_{0}=q\right)$, and write

$$
V=n a^{2}\left(u-u_{0}+e \sin u-e \sin u_{0}\right)
$$

joining to this the equations

$$
\begin{gathered}
r=a(1-e \cos u), \quad r_{0}=a\left(1-e \cos u_{0}\right), \\
\theta-\theta_{0}=\tan ^{-1}\left(\frac{\sqrt{1-e^{2}} \sin u}{\cos u-e}\right)-\tan ^{-1}\left(\frac{\sqrt{1-e^{2}} \sin u_{0}}{\cos u-e}\right),
\end{gathered}
$$

$u, u_{0}, e$ will be functions of $a, r, r_{0}, \theta, \theta_{0}$, and consequently ( $n$ being throughout considered as a function of $a$ ) $V$ will be a function of $a, r, r_{0}, \theta, \theta_{0}$. The function $V$ so expressed as a function of $a, r, r_{0}, \theta, \theta_{0}$ is, in fact, the characteristic function of Sir W. R. Hamilton, and according to his theory we ought to have

$$
d V=\frac{1}{2} n^{2} a\left(t-t_{0}\right) d a+p d r+q d \theta-p_{0} d r_{0}-q_{0} d \theta_{0} .
$$


To verify this, I form the equation

$$
\begin{aligned}
d V= & \frac{1}{2} n a\left(u-u_{0}+e \sin u-e \sin u_{0}\right) d a \\
& +n a^{2}\left[(1+e \cos u) d u-\left(1+e \cos u_{0}\right) d u_{0}\right] \\
& +n a^{2}\left(\sin u-\sin u_{0}\right) d e \\
& +\frac{n a e \sin u}{1-e \cos u}\{d r-(1-e \cos u) d a-a e \sin u d u+a \cos u d e\} \\
& -\frac{n a e \sin u_{0}}{1-e \cos u_{0}}\left\{d r_{0}-\left(1-e \cos u_{0}\right) d a-a e \sin u_{0} d u_{0}+a \cos u_{0} d e\right\} \\
& +n a^{2} \sqrt{1-e^{2}}\left\{d \theta-\frac{1}{\sqrt{1-e^{2}}(1-e \cos u)}\left[\left(1-e^{2}\right) d u+\sin u d e\right]\right. \\
& \left.\quad-d \theta_{0}+\frac{1}{\sqrt{1-e^{2}}\left(1-e \cos u_{0}\right)}\left[\left(1-e^{2}\right) d u_{0}+\sin u_{0} d e\right]\right\}
\end{aligned}
$$

the coefficient of $d u$ on the right-hand side is

$$
\begin{aligned}
& n a^{2}(1+e \cos u)-\frac{n a^{2} e^{2} \sin ^{2} u}{1-e \cos u}-\frac{n a^{2}\left(1-e^{2}\right)}{1-e \cos u} \\
= & n a^{2}\left(1+e \cos u-\frac{1-e^{2}+e^{2} \sin ^{2} u}{1-e \cos u}\right),
\end{aligned}
$$

which vanishes, and similarly the coefficient of $d u_{0}$ also vanishes: the coefficient of de is the difference of two parts, the first of which is

$$
\begin{aligned}
& n a^{2} \sin u+\frac{n a^{2} e \sin u \cos u}{1-e \cos u}-\frac{n a^{2} \sin u}{1-e \cos u} \\
= & n a^{2} \sin u\left(1-\frac{1-e \cos u}{1-e \cos u}\right),
\end{aligned}
$$

which vanishes, and the second part in like manner also vanishes; the coefficient of $d a$ is the difference of two parts, the first of which is

$$
\frac{1}{2} n a(u+e \sin u)-n a e \sin u=\frac{1}{2} n a(u-e \sin u),
$$

and the second is the like function of $u_{0}$; the entire coefficient therefore is

We have therefore

$$
\frac{1}{2} n a\left(u-u_{0}-e \sin u+e \sin u_{0}\right) \text {. }
$$

$$
\begin{aligned}
d V= & \frac{1}{2} n a\left(u-u_{0}-e \sin u+e \sin u_{0}\right) d a \\
& +\frac{n a e \sin u}{1-e \cos u} d r+n a^{2} \sqrt{1-e^{2}} d \theta \\
& -\frac{n a e \sin u_{0}}{1-e \cos u_{0}} d r_{0}-n a^{2} \sqrt{1-e^{2}} d \theta_{0}
\end{aligned}
$$

or what is the same thing,

$$
d V=\frac{1}{2} n^{2} a\left(t-t_{0}\right) d a+p d r+q d \theta-p_{0} d r_{0}-q_{0} d \theta_{0},
$$

the equation which was to be verified.

2, Stone Buildings, March 28, 1856. 